\title{
PRÁTICAS DE SAÚDE DE ESTUDANTES DO GÊNERO MASCULINO DE UM CURSO SUPERIOR EM SAÚDE
}

\author{
Maria Thereza Ávila Dantas Coelho (UFBA)* \\ http://orcid.org/0000-0001-7857-7473 \\ Daniele Machado Pereira Rocha (UFBA)** \\ http://orcid.org/0000-0002-1616-7273 \\ Jorge Luiz Lordêlo de Sales Ribeiro (UFBA)*** \\ http://orcid.org/0000-0002-4186-7394
}

\section{RESUMO}

Atualmente há uma escassez de pesquisas que abordem a temática das práticas de saúde e doença, no sentido coletivo e individual de grupos específicos da população brasileira, em especial a masculina, que possui um histórico de falta de cuidado com a saúde. Objetivo: Identificar e analisar as práticas de saúde e doença de estudantes do gênero masculino, de um curso superior em saúde. Métodos: Os participantes desta pesquisa foram estudantes do gênero masculino, que responderam as perguntas de um questionário semiestruturado. As respostas foram analisadas com base na análise de conteúdo de Bardin. Resultados: Os estudantes responderam, em sua maioria, que utilizavam práticas de atividade física (AT) e alimentação saudável (AS) para manter uma boa saúde e prevenir doenças. Outras práticas de saúde também foram informadas, como práticas integrativas/complementares e religiosas de saúde, porém em pouca quantidade. Concluímos que a maioria das respostas dos alunos (AT e AS) está ligada à concepção de uma boa saúde, podendo significar cuidado, mas também reproduzindo ações reducionistas de saúde. 0 diálogo com o alunado é importante, no sentido da ampliação das práticas de saúde no âmbito coletivo e terapêutico, considerando, inclusive, práticas não convencionais.

Palavras-chave: Autocuidado. Saúde. Estudantes. Universidades.

* Doutora em Saúde Pública pela Universidade Federal da Bahia (UFBA). Professora Associada da Universidade Federal da Bahia (UFBA). Coordenadora do Núcleo de Estudos Interdisciplinares em Saúde, Violência e Subjetividade do Instituto de Humanidades, Artes e Ciências "Professor Milton Santos", da Universidade Federal da Bahia (SAVIS/IHAC/UFBA). E-mail: therezacoelho@gmail.com

** Mestre pelo Programa de Pós-Graduação em Estudos Interdisciplinares Sobre a Universidade da Universidade Federal da Bahia (PPGEISU/UFBA). Participante do Núcleo de Estudos Interdisciplinares em Saúde, Violência e Subjetividade do Instituto de Humanidades, Artes e Ciências "Professor Milton Santos”, da Universidade Federal da Bahia (SAVIS/IHAC/UFBA). E-mail: danielep.rocha@hotmail.com

*** Doutor em Educação pela Universidade Federal da Bahia (UFBA). Professor Associado da Universidade Federal da Bahia e Pesquisador do Núcleo de Estudos Interdisciplinares em Saúde, Violência e Subjetividade do Instituto de Humanidades, Artes e Ciências “Professor Milton Santos”, da Universidade Federal da Bahia (SAVIS/IHAC/UFBA). E-mail: jorgeluizdesales@ gmail.com 


\section{ABSTRACT}

\section{PRACTICES OF HEALTH OF STUDENTS OF GENDER MASCULINE OF A HIGHER COURSE IN HEALTH}

Currently there is a lack of research that addresses the theme of health practices and disease, the collective and individual sense of specific groups in the Brazilian population, especially the masculine that has a historic of lack of care for the health, according to the literature. Objective: To identify and analyze the practices of health of gender students masculine of a higher course in health. Methods: The participants in this study were male students, who answered the questions of a semi-structured questionnaire. The answers were analyzed in the mold content analysis of Bardin. Results: The students responded mostly using physical activity (PA) and healthy eating (HA) practices in maintaining good health and prevent disease. Other promotion practices and prevention were also informed as well as integrative / complementary health and religious practices however in small quantities. Conclusion: The PA and HA are much related to the design of the students of good health, which can mean care, but also plays standard reductionist actions of health. The dialogue with the student body is important, in the direction of broadening of health practices in the collective scope and at the therapeutic context, considering even unconventional practices.

Keywords: Selfcare. Health. University. Students.

\section{RESUMEN \\ LAS PRÁCTICAS DE SALUD DE ESTUDIANTES DEL GÊNERO MASCULINO DE UNO CURSO SUPERIOR EN SALUD}

En la actualidad hay una escasez de investigación que aborda el tema de las prácticas de salud y la enfermedad, el sentido colectivo e individual de grupos específicos de la población, especialmente la masculina, que tiene una carencia de cuidado con la salud. El objetivo: Identificar y analizar las prácticas de salud y enfermedad de estudiantes del género masculino, de uno curso superior en salud. Los métodos: Los participantes eran estudiantes del género masculino, que respondieron a un cuestionario semi estructurado. Las respuestas han sido analizadas sobre la base de análisis de contenido de Bardin. Los resultados: Los estudiantes respondieron que, en su mayoría, usaban prácticas de actividad física y alimentación sana para mantener una buena salud y prevenir enfermedades. También fueron informadas las prácticas religiosas y las prácticas integración / complementario del salud, pero en pequeñas cantidades. Conclusión: La mayoría de las respuestas de los estudiantes está relacionada con el concepción de buena salud y puede significar la atención, pero también prácticas reduccionistas de la salud. El diálogo con el alumnado es importante en el sentido de la expansión de las prácticas de salud en el contexto colectivo y terapéutico, teniendo en cuenta las prácticas incluso no convencionales.

Palabras clave: Autocuidado. Salud. Estudiantes. Universidades. 


\section{Introdução}

Desde o final de 1970, as pesquisas relacionadas à saúde e doença dos homens vêm ascendendo, mesmo que em uma perspectiva norte-americana. Esses estudos, em sua maioria fundamentados na produção da cientista social Raewyn Connell, evidenciam que a masculinidade hegemônica - conjunto de práticas expressas como padrão, exemplar, associadas aos homens - não inclui o comportamento do autocuidado em relação à saúde (CARRARA; RUSSO; FARO, 2009; CONNELL, 1995).

$\mathrm{Na}$ conjuntura latina e brasileira, pesquisas começaram a emergir a partir da década de 1980, com as altas taxas de morbimortalidade entre os homens. Em um desses estudos, feito por Laurenti, Jorge e Gotlieb (2005), percebeu-se que, em todas as idades, a maior taxa de mortalidade acontecia nesse segmento. 0 adoecimento e as mortes ocorridas entre os homens estão ligadas, até os dias atuais, à violência, sejam estes como executores ou vítimas, às neoplasias (a exemplo do câncer de próstata), doenças do aparelho circulatório, digestivo e respiratório (BRASIL, 2009a; PINHEIRO; COUTO, 2008; RODRIGUES; RIBEIRO, 2012).

Frente a essa problemática, o Brasil criou, em 2009, a Política Nacional de Atenção Integral à Saúde do Homem (PNAISH), pensando na vulnerabilidade deste segmento populacional às doenças e no pouco cuidado dos homens com a própria saúde. 0 objetivo principal dessa política é a promoção de ações que auxiliem a compreensão das necessidades de cuidado, na perspectiva social, política, econômica e cultural, visando ao autocuidado, à diminuição das taxas de morbimortalidade e à qualidade de vida individual e coletiva (BRASIL, 2009a; CARRARA; RUSSO; FARO, 2009).

Nessa direção, faz parte do contexto de vida de qualquer população compreender e realizar práticas de saúde coletivas e individuais. As práticas coletivas são caracterizadas como ações necessárias para que os sujeitos obtenham uma boa saúde, extrapolando o individualismo e pautando-se em ações de promoção da saúde para toda a população, de forma equitativa (BUSS, 2000).

As práticas individuais relacionam-se com as práticas de cura, que são as atividades que auxiliam no enfrentamento de doenças, e com as práticas de preservação da própria saúde, que fazem parte do estilo de vida pessoal de cada sujeito e podem ser influenciadas por fatores externos, como a mídia e os grupos em que o sujeito esteja inserido, envolvendo, neste sentido, aspectos educacionais, familiares e culturais, sendo elas necessárias para a preservação de vida (ACIOLI, 2006). Segundo Escobar e Pico (2013), as práticas de autocuidado são atividades executadas por motivações próprias, para si mesmos, com livre escolha de equipamentos e materiais, cujo objetivo é preservar a vida e a saúde. Nesse âmbito temos também as práticas terapêuticas biomédicas e as práticas integrativas e complementares, que promovem o tratamento/cura de doenças e contribuem para o bem-estar corporal, mental e espiritual; bem como as práticas religiosas/ espirituais, realizadas em igrejas, grupos de apoio e centros espirituais, que contribuem para a saúde e a cura de doenças (ACIOLI, 2006; TROVÓ; SILVA, 2002).

Nesse contexto, as práticas de saúde podem ser categorizadas do seguinte modo: práticas coletivas de promoção da saúde; práticas individuais de preservação da saúde (estilo de vida); práticas preventivas (de evitação do surgimento das doenças) e práticas terapêuticas. Essas práticas podem ser biomédicas, integrativas e complementares e/ou religiosas/ espirituais. Considerando a importância e a escassez de estudos sobre práticas de saúde de estudantes homens universitários da área da saúde, bem como os possíveis benefícios que pesquisas desta natureza podem trazer para a temática da saúde e do adoecimento de grupos específicos, o objetivo deste artigo é identificar e analisar tais práticas entre estudantes do 
gênero masculino, ingressantes no primeiro semestre do Bacharelado Interdisciplinar em Saúde da Universidade Federal da Bahia (BIS/ UFBA).

\section{Promoção da saúde e práticas coletivas de saúde}

A expressão "promover saúde" está ligada à ideia de impulsionar, originar, gerar medidas que não se dirijam a doenças específicas, mas que visem o aumento da saúde e do bem-estar da população de forma equitativa, buscando justiça social, evitando situações injustas e desnecessárias. 0 conceito de promoção da saúde está diretamente relacionado com os Determinantes Sociais da Saúde (DSS), que compreendem os fatores sociais, econômicos, culturais, étnico-raciais, psicológicos e comportamentais que influenciam as condições de vida da população (BUSS, 2000; BUSS; PELLEGRINI FILHO II, 2007; CZERESNIA, 2003).

Segundo Czeresnia (2004), o termo promoção da saúde foi primeiramente mencionado por Henry Sigerist, médico historiador que, em 1945, destacou quatro funções essenciais na medicina: promoção da saúde, prevenção de doença, reabilitação e cura. Leavell e Clark, por sua vez, em 1976, conceberam a medicina preventiva, com a história natural das doenças, definindo esta como resultante de todas as interações do agente, hospedeiro e meio ambiente que afetam o processo global e o desenvolvimento do corpo. Esse modelo, no entanto, se baseava na explicação do processo de adoecimento e não dava conta de aspectos crônicos, ou seja, das doenças de longa duração. A partir dessa ineficácia desse modelo, o conceito de promoção da saúde se desenvolveu em suas relações com o modelo dos determinantes sociais da saúde, de Dahlgren e Whitehead, que compreende a saúde a partir das condições de saúde da população, sendo esta uma referência importante para o estudo da promoção da saúde (BUSS, 2000; CZERESNIA, 2004).
Diversas reuniões para debater as ações de promoção da saúde têm sido realizadas pelo mundo. A mais importante delas, e crucial para a criação de novos encontros, foi a Conferência de Ottawa, ocorrida no Canadá em 1986, que estabeleceu cinco elementos essenciais para a operacionalização das práticas de promoção da saúde, sendo eles: implementação de políticas públicas saudáveis; criação de ambientes saudáveis favoráveis à saúde; reorientação dos serviços de saúde; reforço da ação comunitária; e desenvolvimento das habilidades pessoais da população, a exemplo do empowerment, que se caracteriza por proporcionar aos sujeitos um conhecimento sobre a saúde, tanto de forma coletiva quanto individual, de modo a que eles/elas possam enfrentar e buscar, junto aos órgãos competentes, resolver problemas de saúde em sua comunidade, residência ou local de estudo ou trabalho (CARVALHO, 2004; HEIDMANN et al, 2006; SICOLI; NASCIMENTO, 2003).

No Brasil, também em 1986 aconteceu a VIII Conferência Nacional de Saúde, que convocou atores sociais, políticos e profissionais da área da saúde para discutirem políticas públicas de saúde, um programa de reforma sanitária, bem como a proposta de criação do Sistema Único de Saúde (SUS), na perspectiva de que a saúde é um direito da população e um dever do Estado (BERTOLOZZI; GRECO, 1996). Após a criação do SUS, em 1990, e amplas discussões, foi aprovada, em 2006, a Política Nacional de Promoção da Saúde (PNPS), cujo objetivo é "promover a qualidade de vida e reduzir vulnerabilidade e riscos à saúde relacionados aos seus determinantes e condicionantes - modos de viver, condições de trabalho, habitação, ambiente, educação, lazer, cultura, acesso a bens e serviços essenciais" (BRASIL, 2006a, p. 19). Essa política abrange práticas coletivas e intersetoriais, de promoção da saúde e prevenção de doenças, ligadas a políticas públicas saudáveis, que envolvem alimentação saudável, atividade corporal, prevenção e controle do tabagismo, prevenção da violência, redução da 
morbimortalidade por acidentes de trânsito, dentre outros aspectos.

Especificamente em relação aos homens, embora, em 2009, tenha sido criada a PNAISH, não vem sendo debatida, com profundidade, nessa política, sua operacionalização. No Plano de Ação Nacional 2009-2011, ligado à PNAISH, foram mencionadas estratégias e ações de promoção da saúde e prevenção de doenças, que levaram à execução de medidas voltadas para a educação de diferentes formas de pensar e agir, articuladas com movimentos sociais, integrando homens de todos os segmentos e orientações, bem como estimulando novas práticas de cuidado com a saúde (BRASIL, 2009a, 2009b). Embora o conceito de promoção da saúde também esteja ligado a ações individuais, relacionando-se, de acordo com o modelo de Dahlgren e Whitehead, com o estilo de vida da população, essas ações podem ser influenciadas por fatores externos, como a mídia, falta de acesso a alimentos saudáveis e a espaços de entretenimento, dentre outros, fazendo com que o escopo do termo promoção da saúde esteja diretamente ligado à saúde coletiva.

\section{As práticas individuais de saúde}

A partir do exposto, percebemos que existem duas linhas conceituais sobre a promoção da saúde: as práticas coletivas ligadas às condições de vida da população e o estilo de vida individual. 0 conceito de estilo de vida vem sendo debatido desde 1974, quando o tema foi discutido no Canadá, gerando o Informe Lalonde, segundo o qual a mudança de comportamentos e de estilo de vida, ou seja, a promoção da saúde, é da responsabilidade dos indivíduos, sendo os mesmos culpabilizados pelo desvio das práticas consideradas saudáveis (BUSS, 2000).

Segundo Nahas, Barros e Francalacci (2000), estilo de vida saudável significa um conjunto de práticas diárias que conscientemente o sujeito realiza para manter uma qualidade de vida. As ações realizadas podem mudar ao longo dos anos e/ou sofrer influência de fatores tanto hereditários quanto de idade, cultura e ambiente onde se vive. Matos e Sousa-Albuquerque (2006) assinalam que a falta de exercício físico, o hábito de fumar, a utilização de drogas lícitas e/ou ilícitas, bem como acidentes e práticas sexuais desprotegidas formam um conjunto de práticas que podem possibilitar o adoecimento.

A inexistência de práticas individuais de saúde pode ser associada aos altos índices de morbimortalidade entre homens, em sua maioria devido a fatores externos (violência), neoplasias, doenças do aparelho digestivo e acidentes, fruto, na maioria das vezes, da falta de cuidado (BRASIL, 2009a; NAHAS; BARROS; FRANCALACCI, 2000). Segundo Nahas (1996), existem três características relacionadas ao estilo de vida individual que interferem ou contribuem para a saúde: o nível de estresse, a nutrição e as atividades físicas realizadas. Esse autor acrescentou a elas fatores como relacionamentos e a não utilização de drogas, formando um pentágono com os seguintes fatores: nutrição, estresse, comportamento preventivo, atividade física e relacionamentos. É importante destacar que a Política de Alimentação e Nutrição do Ministério da Saúde define que o que compõe uma alimentação saudável são alimentos munidos de água, carboidratos, proteínas, lipídios, vitaminas, fibras e minerais, sendo esses vitais para o bom funcionamento do organismo (BRASIL, 2006b).

Nessa direção, e abordando os fatores externos que podem influenciar uma alimentação saudável, Nahas, Barros e Francalacci (2000), em consonância com o Guia Alimentar para a População Brasileira, do Ministério da Saúde (BRASIL, 2006b), condenam os veículos de comunicação quando estes expõem a população a dietas que se dizem eficazes, com baixos nutrientes, que limitam a quantidade de ingestão de alimentos, provocando, assim, prejuízos à saúde. A informação faz parte de uma vida saudável e o que comemos e bebemos não é somente uma predileção de cada sujeito, mas sofre a ingerência do ambiente social, midiá- 
tico, familiar, dentre outros (BRASIL, 2006b; GOMES, 2010).

Como mencionado anteriormente, o estilo de vida pode sofrer a influência de outros fatores, sendo um deles a mídia. Connell e Messerschmidt (2013) citam o papel dos meios midiáticos como importantes na divulgação de imagens representativas. Com relação aos homens, essas imagens geralmente estão ligadas aos esportes e aos comportamentos de risco. Esses autores mostram ainda que se tem tentado abordar mais os determinantes sociais da saúde na mídia, mas esta continua priorizando a associação de aspectos de cunho sexual/esportivo às práticas de saúde masculinas.

Hoff (2004) cita que a mídia é capacitada para difundir imagens que permeiam o imaginário coletivo e que são frequentemente aceitas. Essa autora afirma ainda que, no tocante ao corpo masculino, as concepções de atividade física estão direcionadas ao culto ao corpo, à moda e à beleza. Com isso, o interesse pelo público masculino nessa atividade aumenta cada dia mais, pois associada a ela é veiculada a imagem de um corpo ideal, saudável, socialmente aceitável e desejável.

No entanto, embora a mídia tenha a capacidade de motivar as pessoas a reverem suas práticas de saúde, tanto de forma positiva quanto negativa, não pode ser colocada em um patamar de poder único e capaz de alienar toda uma população. É necessário que se compreenda que cada sujeito é capaz de criticar e discordar do conteúdo acessado e que seu contexto sócio-político-cultural interfere nas suas escolhas e na absorção das informações que lhe são apresentadas.

Cabe ressaltar que a adoção de práticas individuais de saúde é importante, pois reduz os índices de gordura corporal, sedentarismo, bem como proporciona bem-estar no cotidiano de vida e de trabalho, auxiliando no controle da depressão, ansiedade, tabagismo e outras drogas. Também previne e auxilia no tratamento de doenças cardiovasculares, diabetes, osteoporose, neoplasias, hipertensão arterial e estresse, contribuindo para o aumento da expectativa de vida de homens e mulheres (ALVES et al, 2005; SOUZA JUNIOR; BIER, 2008).

\section{Práticas integrativas/ complementares e religiosas/ espirituais de saúde}

As Práticas Integrativas/Complementares (PIC) de saúde são definidas como um grupo de práticas que, na atualidade, não fazem parte da medicina convencional - esta considerada por utilizar em suas ações agentes farmacológicos para tratar as doenças. As PIC utilizam outros recursos terapêuticos e abrangem ações de apoio ao sujeito na prevenção e/ou no tratamento, seja ele espiritual, corporal ou mental, promovendo saúde e prevenindo doenças. Atualmente foram identificadas mais de 231 terapias tradicionais distribuídas pelo mundo, a exemplo de países do continente africano, onde não há facilidade no acesso a medicamentos farmacêuticos. Desse modo, as PIC contribuem para a cura de doenças e para a qualidade de vida da população (BRASIL, 2006c; MORALES; MIN; TEIXEIRA, 2015; TROVÓ; SILVA, 2002).

A discussão mais formal acerca das PIC iniciou-se na Conferência Mundial de Alma-Ata, ocorrida em 1978 na antiga União Soviética. Nesse debate, a Organização Mundial da Saúde (OMS) enfatizou a necessidade de uma política que abordasse práticas medicinais populares em benefício da população. No Brasil, as PIC começaram a ser discutidas na $8^{\circ}$ Conferência Nacional de Saúde, em 1986, obtendo crescente visibilidade. Em 2006, a Política Nacional de Práticas Integrativas e Complementares no Sistema Único de Saúde (PNPIC/SUS) foi publicada, reconhecendo oficialmente cinco dessas práticas - acupuntura, fitoterapia, homeopatia, termalismo social/crenoterapia e medicina antroposófica (BRASIL, 2006c).

A PNPIC tem o objetivo de intervir na prevenção de doenças e na promoção da saúde, com ações que visem à preservação e recupe- 
ração dos sujeitos de uma forma humanizada e direcionada para a integralidade da atenção aos usuários do sistema de saúde. Dessa forma, a política é fortalecida pelos princípios norteadores do SUS: universalidade, equidade e integralidade. Além disso, a PNPIC visa o aumento do número de profissionais capacitados e a melhora na qualidade do serviço, contribuindo assim para uma maior aderência da população a essas práticas no SUS (BRASIL, 2006c).

Salientamos que o reconhecimento e a utilização das PIC contribuem para a melhoria da saúde da população, incluindo a parcela que ainda não possui acesso ao serviço primário, seja pela falta de recurso financeiro, seja pela distância existente entre sua residência e os postos de saúde. Em alguns casos, a eficácia desses tratamentos pode ser equivalente à dos métodos convencionais. Apesar de eles enfrentarem resistência de aceitação e de divulgação, quando conhecidos facilitam a vida e a saúde da população (BRASIL, 2006c; TROVÓ; SILVA, 2002).

No que concerne às práticas religiosas/ espirituais ligadas à saúde, embora não exista uma política pública que as englobe, estas são amplamente discutidas na antropologia como importantes no processo de cura e bem-estar de seus seguidores. Os antropólogos afirmam que, em comparação com a medicina convencional (que muitas vezes não explica o motivo do adoecimento no contexto de vida do sujeito), as práticas de saúde realizadas pelas religiões inserem o contexto sociocultural das pessoas que as buscam (COSTA-ROSA, 2008).

Existem no Brasil inúmeros ritos religiosos que possuem caráter de cura, em igrejas evangélicas, terreiros de candomblé, centros espíritas, comunidades indígenas e quilombolas, dentre outras, todas com suas especificidades, seja com o uso das plantas nas religiões de matriz africana e indígena, com as cirurgias espirituais realizadas no espiritismo, como também com as orações feitas nas igrejas evangélicas, ou com as rezas realizadas por rezadeiras(os) em quilombos e interiores do país. Em todas essas práticas há pessoas que buscam mudanças no seu corpo, de ordem física, mental ou espiritual (ANDRADE, 2007; BOTELHO, 2010; COSTA-ROSA, 2008; PIETRUKOWICZ, 2001; VIDAL, 2012).

A religiosidade produz na população uma ligação com o divino e, para muitas pessoas, esse elo proporciona qualidade de vida. A sensação de paz, restauração da autoestima, esperança e enfrentamento dos problemas cotidianos são necessidades que muitos sujeitos almejam satisfazer e que o conseguem através dessas práticas. Tanto as práticas de saúde religiosas/espirituais quanto as integrativas/ complementares possibilitam soluções para problemas que a medicina convencional pode não possuir explicações (ANDRADE, 2007; TROVÓ; SILVA, 2002).

\section{Metodologia}

Este estudo é parte do resultado de uma dissertação de mestrado (ROCHA, 2017) e integra uma pesquisa maior, que investiga as concepções e práticas pessoais e profissionais ligadas a processos de saúde e doença, através de um questionário semiestruturado contendo 33 perguntas acerca desta temática (ROCHA, 2017). Esse instrumento foi aplicado a 212 estudantes recém-ingressos no Bacharelado Interdisciplinar em Saúde (BIS) da Universidade Federal da Bahia (UFBA), no início do primeiro semestre letivo de 2014, dos quais 79 eram do gênero masculino e 76 participaram desta pesquisa, com idade que variava entre 17 e 61 anos.

Foram selecionadas, do questionário, oito perguntas referentes às práticas de saúde desses alunos: Que ações você considera importantes para manter a saúde?; Quais dessas ações você realiza para manter a sua saúde?; Que ações você considera importantes para prevenir doenças?; Quais dessas ações você já realizou para prevenir doenças?; 0 que você faz quando está doente?; Sobre sua alimentação, assinale seus hábitos; Agora falaremos sobre 
seu hábito de praticar atividade física; Qual(is) são as práticas integrativas/complementares e/ou religiosas que você utiliza? Os sujeitos participantes do estudo responderam ou não a todas as perguntas acima citadas, de forma livre e sem identificação.

Os dados coletados foram analisados com base na análise de conteúdo, focando na análise temática de Bardin (2009), que a define como uma união de técnicas visando alcançar o detalhamento do conteúdo que, neste caso, se caracteriza pelas respostas dos sujeitos, interpretadas à luz da revisão de literatura. Desse modo, a análise e interpretação dos dados foram feitas a partir dos seguintes passos e da seguinte forma: as respostas das quatro primeiras perguntas, que eram abertas, foram digitadas no processador de texto Microsoft Word $®$ e analisadas de forma qualitativa. As demais perguntas, de natureza objetiva, continham a opção de assinalar um ou mais itens e foram analisadas no editor de planilhas $\mathrm{Mi}$ crosoft Office Excel@, sendo contabilizadas em número absoluto de estudantes e porcentagem.

As respostas dos participantes da pesquisa foram distribuídas em quatro categorias gerais, algumas com subdivisões, a saber: práticas individuais de preservação da saúde, que comportam as subcategorias alimentação saudável, atividade física e outras práticas; práticas coletivas de promoção da saúde, sem subcategorias; práticas de prevenção de doenças, com as subcategorias alimentação saudável, atividade física e outras práticas; práticas terapêuticas, com as subcategorias práticas médicas/profissionais, automedicação, práticas integrativas/complementares e tratamentos religiosos. Essas categorias e subcategorias foram construídas a partir das respostas dadas pelos estudantes.

O estudo foi aprovado pelo Comitê de Ética em Pesquisa (CEP) da Escola de Enfermagem da UFBA, sob o $\mathrm{n}^{\circ}$ 741.187, respeitando os dispositivos da Resolução no 466/13 do Conselho Nacional de Saúde sobre Pesquisa com Seres Humanos. Os estudantes participantes assinaram um termo de consentimento livre e esclarecido (TCLE) de participação. Declaramos que não há conflito de interesses na elaboração do trabalho.

\section{Resultados e discussão}

Os resultados obtidos com o questionário, no tocante aos dados sociodemográficos, demonstraram que $86 \%$ dos estudantes eram solteiros; $37 \%$ católicos; $45 \%$ estudaram em escola pública e $45 \%$ em escola privada; $84 \%$ não possuíam outra formação acadêmica, ao passo que $16 \%$ possuíam. Todos os estudantes responderam ser do gênero masculino. Tomarse-á como referência para a apresentação dos resultados e discussão as categorias descritas na metodologia. A letra S será utilizada no início das respostas, indicando cada sujeito que respondeu ao questionário.

\section{Práticas coletivas de promoção da saúde}

Do total de participantes desta pesquisa, apenas seis estudantes citaram aspectos relacionados a essas práticas. Apesar disso, as respostas alocadas nesta categoria indicam que esses alunos entendem que a saúde vai além da responsabilidade do indivíduo. Tal achado sugere uma aproximação dos mesmos com o cuidado com os outros, além do autocuidado (MINAYO, 2010).

Saneamento básico adequado, educação de qualidade, informação, atendimento ao indivíduo de forma universal, infraestrutura. (S22).

Políticas públicas afirmativas, efetivas e resolutivas, que promovam ações adequadas para proporcionar o bem-estar social. (S26).

As respostas destacadas acima demonstram uma concepção ampliada de saúde, relacionando-se com a $3^{\text {a }}$ camada do modelo de Dahlgren e Whitehead dos determinantes sociais da saúde, que indicam o trabalho, renda, educação, lazer, saneamento, agricultura, habitação 
e serviços de saúde como essenciais para a promoção da saúde da população (BUSS; PELLEGRINI FILHO II, 2007).

Todos os estudantes que responderam ao questionário são do $1^{\circ}$ semestre do BIS/UFBA, sendo que $16 \%$ responderam que já possuem outra graduação concluída. Não temos a informação do curso realizado anteriormente, mas podemos associar as respostas referentes à promoção da saúde a informações adquiridas possivelmente com essa formação, de forma direta ou indireta.

Para além de toda a literatura mencionada acima, Gomes (2010) cita, em seu livro sobre a saúde do homem, que a informação é essencial para uma boa saúde. Desse modo, se faz necessário colocar em prática mais estudos relativos à promoção da saúde nos currículos dos cursos de saúde, bem como nas escolas, para que mais estudantes tenham acesso à perspectiva da promoção da saúde, que extrapola a noção da saúde centrada na ausência de doença.

\section{Práticas individuais de preservação da saúde}

Analisando as respostas categorizadas como práticas de preservação da saúde e suas subdivisões, percebe-se que tais práticas circulavam, em sua grande maioria, entre a atividade física e a alimentação saudável. Ao todo foram 64 respostas de estudantes para práticas de atividades físicas, que incluíam também a palavra "esporte", mas sem especificação do tipo. Houve 55 respostas correspondentes à alimentação saudável. Algumas delas estão destacadas a seguir:

Ter uma alimentação saudável [...] Prática de atividades físicas. (S2).

Manter uma boa alimentação [...] Realizar atividades físicas diárias. (S63).

Podemos observar e relacionar o grande número de respostas referentes à atividade física/exercício físico e alimentação saudável a uma mudança de discurso com relação à ne- cessidade de preservação da saúde. 0 estilo de vida desses discentes pode ou não estar sendo influenciado pelo desejo de uma melhora no desempenho físico, o que, associado a uma dieta balanceada, proporciona um corpo que é difundido pela mídia como ideal, conforme o contexto cultural e o meio onde estão inseridos. Sendo assim, o binômio atividade física e alimentação é importante tanto para a melhoria da saúde, como para o conjunto de práticas necessárias para o pertencimento a um grupo social.

Com relação às outras respostas de preservação da saúde apresentadas pelos estudantes, incluídas na subcategoria "outras práticas", obtivemos respostas relativas ao hábito de lavar as mãos, ao uso de protetor solar, consumo de líquidos, higiene pessoal, uso restritivo de fumo e álcool, evitação do estresse, boa noite de sono, descanso, comunhão com Deus, equilíbrio mental e namoro com responsabilidade. Todas essas respostas totalizaram 74. Alguns fragmentos delas podem ser observados a seguir:

\section{[...] higiene [...] prevenção [...]. (S2).}

Namorar nosso companheiro com responsabilidade. (S33).

Ao analisar as respostas dos alunos, destacamos a presença de uma compreensão mais alargada sobre as práticas de preservação da saúde, pois alguns deles citam, além do que é destacado na mídia, o relacionamento com seus parceiros, o sono e o descanso também como necessários para uma boa saúde. As respostas também estão relacionadas com o pentáculo do bem-estar, uma vez que incluem o uso do protetor solar - indicado para evitar a exposição com excesso ao sol e prevenir câncer de pele e envelhecimento antecipado -, uso de preservativos - a fim de não se contaminar com doenças sexualmente transmissíveis (DST) -, bem como o não uso de drogas lícitas e/ou ilícitas, que podem provocar sérios problemas sociais, comportamentais e psicológicos (BUSS; PELLEGRINI FILHO II, 2007; NAHAS, 1996; NAHAS; BARROS; FRANCALACCI, 2000). 


\section{Práticas de prevenção de doenças}

As práticas de prevenção de doenças, apresentadas pelos alunos, foram praticamente as mesmas práticas individuais de preservação da saúde: alimentação saudável e atividade física. Nesse sentido, é importante destacar que os estudantes relacionam mais a preservação da saúde à prevenção de doenças e vice-versa. Tal aspecto pode ser explicado pelo fato de $84 \%$ dos discentes não possuírem outra graduação concluída e não ser comum nas escolas haver explicações sobre a diferença entre práticas de prevenção de doenças, que implicam o conhecimento epidemiológico para se conhecer e combater os riscos antes que se adoeça, e práticas de promoção da saúde, que podem ser individuais ou coletivas (BUSS, 2000).

As demais práticas de prevenção apresentadas pelos alunos foram agrupadas na subcategoria "outras práticas", referentes à higiene, ingestão de líquidos, uso restritivo de álcool e outras drogas, vacinação, qualidade do sono, equilíbrio espiritual, comunhão com Deus, descanso e equilíbrio corporal. A seguir, estão algumas dessas respostas:

[...] Prevenção e orientações de doenças [...]. (S44).

[...] Boa higienização com tudo que lidamos, vacinação, métodos preventivos [...]. (S70).

É importante destacar que, para os participantes desta pesquisa, essas práticas fazem parte de um conjunto de ações importantes para prevenir doenças, que envolvem religião, relacionamentos saudáveis e cuidados com o corpo. Essas ações podem evitar doenças como cânceres, arboviroses - doenças transmitidas por mosquitos, a exemplo da dengue -, depressão, tabagismo, hipertensão, dentre outras. Elas contribuem para a prevenção de doenças tanto em homens quanto em mulheres, já que não fazem distinção de gênero, nem de etnia (ALVES et al, 2005; SOUZA JUNIOR; BIER, 2008).
Nas subcategorias "atividade física" e "alimentação saudável”, obteve-se o maior número de respostas dos estudantes, ou seja, 38 e 37, respectivamente.

Praticar exercícios físicos [...] manter uma alimentação balanceada. (S2).

Fazer atividade física [...] manter boa alimentação. (S49).

O conjunto "atividade física e alimentação saudável" novamente congrega o maior número de respostas dos estudantes e corrobora com outras questões do questionário, em que percebemos também a independência dos estudantes na prática da atividade física. Dos discentes pesquisados, 49\% disseram praticar tal atividade, mesmo sem orientação. No que concerne ao hábito alimentar desses discentes, percebemos que, de forma geral, as respostas com números significativos estão relacionadas ao consumo de mais de 2 litros de água por dia (71\%), consumo de verduras e frutas diárias (53\% e 51\%, respectivamente) e mínimo de 3 refeições por dia (87\%).

Essas respostas podem ser associadas ao guia alimentar para a população brasileira, do Ministério da Saúde, que informa que, para se obter uma boa saúde, é necessária a ingestão de pelo menos três refeições por dia: o café da manhã, o almoço e o jantar. Os alimentos considerados importantes são aqueles que possuem concentração de carboidratos, como arroz, massas, pães, raízes (como a mandioca) e tubérculos (como a batata). Também devem ser incluídas na dieta frutas, legumes e verduras, bem como alimentos ricos em proteínas, como cereais, castanhas e sementes. 0 guia alimentar, em consonância com outros autores, reprova dietas milagrosas veiculadas pela mídia por concordar que estas, em sua maioria, não incluem os alimentos necessários para uma boa alimentação (BRASIL, 2006b; NAHAS; BARROS; FRANCALACCI, 2000).

\section{Práticas terapêuticas}

A última categoria, nomeada de práticas terapêuticas, teve como subcategorias: prá- 
ticas médicas/profissionais, automedicação, práticas integrativas/complementares e tratamentos religiosos. As respostas nos deram suporte para compreender se há a utilização dessas práticas entre os estudantes. Desse modo, analisando as respostas dessa categoria, percebemos que $93 \%$ procuram por médicos e/ou profissionais da área da saúde, 71\% dos discentes utilizam a automedicação quando estão doentes, $32 \%$ recorrem às práticas integrativas/complementares e $20 \%$ aos tratamentos religiosos.

As respostas concernentes à busca por profissionais de saúde (93\%) nos permitem compreender como o papel do médico e dos profissionais ligados à área da saúde tem um peso significativo na vida da população. Apesar disso, a visão biomédica, que reduz o sujeito à doença, frequentemente embasa o atendimento aos mesmos e, com isso, os usuários não se sentem acolhidos (MINAYO, 1988). Há uma necessidade de humanização da prática desses profissionais, para que exista uma escuta dos sujeitos, com acolhimento, foco na atenção primária e capacitação para que trabalhem de forma interdisciplinar, aliando seu saber ao popular. Embora a maioria dos estudantes afirme procurar os serviços de saúde, a literatura aponta que muitos homens se distanciam desses serviços por não se sentirem abrigados, por fatores como o horário de atendimento, propagandas relacionadas à saúde feminina e despreocupação com o cuidado (GOMES, 2012; HEIDMANN et al, 2006).

No que se refere à ingestão de medicamentos sem prescrição/orientação de profissionais habilitados da área da saúde, esta é considerada pela Agência Nacional de Vigilância Sanitária (ANVISA) como comportamento de risco, pois pode levar à intoxicação. Ressalta-se também que o uso de medicamentos sem prescrição acarreta uma redução de pacientes nos hospitais/postos de saúde e que parte da população depende diretamente do SUS. Muitas vezes, acessar o sistema público de saúde por simples sintomas que poderiam ser aliviados com a automedicação torna-se um transtorno para a população. Não podemos, no entanto, considerar que essa seja a realidade dos estudantes desta pesquisa, por não possuirmos dados que revelem o motivo pelo qual esses discentes utilizam a automedicação quando estão doentes.

Nas respostas sobre as práticas integrativas/complementares, $32 \%$ dos alunos responderam que as utilizam quando doentes. Complementando esse dado, temos as respostas da pergunta referente a quais dessas práticas são utilizadas por eles. Observamos que o maior número de respostas foi relativo à massagem (20\%), seguido da homeopatia (15\%) e da fitoterapia (13\%).

Embora as respostas apresentadas estejam em menor quantidade, elas fazem parte dos resultados obtidos com relação às práticas de saúde e podem representar também uma falta de conhecimento ou aderência desses estudantes a elas. Estudos de Külkamp e outros (2007) afirmam que a falta de divulgação e inserção desse tema na universidade contribui para a desinformação e não utilização dessas práticas. Em outro estudo com alunos de enfermagem, Gavin, Oliveira e Silva (2010) citam que os/as estudantes têm conhecimento dessas práticas, embora não as adotem ou desconheçam a regulamentação de sua utilização pelo Conselho Federal de Enfermagem (COFEN). Na UFBA existe a Liga Acadêmica de Práticas Integrativas e Complementares em Saúde (LAPICS), que fomenta discussões acerca dessa temática, podendo os discentes, assim, no decorrer do curso, buscar mais informações sobre as mesmas, aumentando a frequência de seu uso.

Referente aos tratamentos religiosos, $20 \%$ dos estudantes respondeu que os utilizam. Esse dado é confirmado pelas respostas à pergunta acerca de quais práticas religiosas eles utilizavam, já que a soma destas práticas - referentes à cirurgia espiritual (8\%), rezas (5\%) e outros rituais de cura (7\%) - também totaliza $20 \%$ das respostas. Esses dados, embora com menor frequência, muito têm a ver com 
a presença da religiosidade em nossa cultura, com a mestiçagem entre os povos indígenas, europeus e africanos, que trouxeram consigo práticas e rituais religiosos de cura. Os dados sociodemográficos dos estudantes mostram que muitos são católicos, outros são evangélicos e espíritas, o que faz com que possam realizar práticas de banho de folhas, orações e rezas, muito utilizadas nessas religiões para descarregar as energias negativas, retomar o equilíbrio, purificar o corpo, dentre outros objetivos (BOTELHO, 2010; REIS, 2007).

\section{Considerações finais}

Embora diversos estudos apontem para um distanciamento dos homens com o cuidado da própria saúde, percebemos que, de uma maneira geral, esta pesquisa diverge do que vem sendo exposto pela literatura. Sabe-se que o universo estudado não é representativo da população como um todo, mas nem por isso estes dados deixam de ser relevantes. Eles apontam, justamente, para a necessidade de um estudo mais amplo com toda a população. As respostas indicam que os alunos pesquisados, do gênero masculino, compreendem a necessidade de autocuidado e adotam práticas de saúde.

Nessa direção, percebemos, na análise das respostas dos discentes, a utilização de hábitos que preservam a saúde e previnem doenças, ligados à alimentação saudável, e que estão de acordo com o guia alimentar para a população brasileira, proposto pelo Ministério da Saúde. Em tempos de ampla correria cotidiana, em que dietas "milagrosas" para a obtenção de um corpo ideal e diversas propagandas de lanches rápidos são oferecidos como refeições, é importante que os alunos tenham e mantenham uma alimentação rica em proteínas, água, carboidratos, vitaminas, fibras e minerais.

No que concerne à prática de atividades/ exercícios físicos, resposta utilizada com frequência pelos discentes para obter uma boa saúde, esta pode estar associada tanto à prática recomendada pela OMS, a fim de prevenir doenças e melhorar o bem-estar, como pode ser realizada com o objetivo de inserção social, de modo a alcançar corpos normatizados para conseguir sucesso afetivo, sexual e profissional.

Também percebemos outras práticas de cuidado à saúde em menor quantidade, como as práticas integrativas/complementares e religiosas/espirituais, o que revela a presença de elementos ligados a outras racionalidades terapêuticas, bem como à religião/espiritualidade desses estudantes, à sua cultura e meio onde estão inseridos. Essas outras formas de cuidado, que são pouco exploradas cotidianamente, demonstram que o cuidado com a saúde vai além da busca por profissionais da área e da automedicação.

Perante as poucas respostas referentes à promoção da saúde coletiva da população, torna-se indispensável a reflexão sobre o que pensamos sobre o objeto "saúde". Esta pesquisa mostra que muitos discentes concebem a saúde a partir da perspectiva prevencionista da doença, focalizando, nessa direção, os hábitos individuais. Diante disso, é importante a necessidade da inclusão do tema "promoção da saúde", no âmbito coletivo, na formação de todos(as) os/ as alunos(as), pois os seus efeitos vão além do cuidado com a própria saúde. A noção de saúde coletiva na vida deles poderá influenciar a vida de suas (seus) companheiras(os), familiares, amigos(as) e sociedade em geral, melhorando inclusive a visão ainda limitada, existente no imaginário coletivo, com relação aos homens e seus cuidados à saúde. 0 diálogo na universidade possibilita tais mudanças, provocando uma visão mais crítica sobre as práticas de saúde e doença, de modo a ressignificá-las, proporcionando melhorias no âmbito familiar, social, econômico, político, educacional e de saúde.

\section{Referências}

ACIOLI, Sonia. Sentidos e práticas de saúde em grupos populares e a enfermagem em saúde pública. Revista Enfermagem, Rio de Janeiro, v. 14, n. 1, p. 21-26, 2006. Disponível em: http://goo. gl/Z9HiLy. Acesso em: 31 mar. 2016. 
ALVES, João Guilherme Bezerra. Et al. Prática de esportes durante a adolescência e atividade física de lazer na vida adulta. Revista Brasileira de Medicina do Esporte, Niterói, RJ, v. 11, n. 5, p. 291294, 2005. Disponível em: http://goo.gl/ppQSP5. Acesso em: 05 ago. 2015.

ANDRADE, Débora. Uma breve reflexão sobre a importância do resgate da função terapêutica religiosa através das práticas de cura. Revista Eletrônica Inter-Legere, Rio Grande do Norte, n. 2, p. 1-11, 2007. Disponível em: http://goo.gl/kGB4Ll. Acesso em: 29 mar. 2016.

BARDIN, Laurence. Análise de conteúdo. 4. ed. Lisboa: Edições 70, 2009.

BERTOLOZZI, Maria Rita; GRECO, Rosangela Maria. As políticas de saúde no Brasil: reconstrução histórica e perspectivas atuais. Revista Escola de Enfermagem da USP, São Paulo, v. 30, n. 3, p. 380398, 1996. Disponível em: http://goo.gl/9pA2z8. Acesso em: 29 mar. 2016.

BOTELHO, Pedro Freire. O segredo das folhas e os rituais de cura na tradição afro-brasileira. In: ENCONTRO DE ESTUDOS MULTIDISCIPLINARES EM CULTURA (ENECULT), 4, 2010, Salvador. Anais [...]. Salvador: FACOM/UFBA, 2010. p. 1-12.

BRASIL. Ministério da Saúde. Política Nacional de Promoção da Saúde. Brasília, DF, 2006a.

BRASIL. Ministério da Saúde. Secretaria de Atenção à Saúde. Coordenação Geral da Política de Alimentação e Nutrição. Guia alimentar para a população brasileira: promovendo a alimentação saudável. Brasília, DF, 2006b.

BRASIL. Ministério da Saúde. Portaria no 971, de 03 de maio de 2006. Aprova a Política Nacional de Práticas Integrativas e Complementares (PNPIC) no Sistema Único de Saúde. Brasília, DF, 2006c.

BRASIL. Ministério da Saúde. Secretaria de Atenção à Saúde. Departamento de Ações Programáticas e Estratégicas. Política Nacional de Atenção Integral à Saúde do Homem: princípios e diretrizes. Brasília, DF, 2009a.

BRASIL. Ministério da Saúde. Secretaria de Atenção à Saúde. Departamento de Ações Programáticas e Estratégicas. Plano de Ação Nacional 2009-2011 da Política Nacional de Atenção Integral à Saúde do Homem. Brasília, DF, 2009b.

BUSS, Paulo Marchiori. Promoção da saúde e qualidade de vida. Ciência \& Saúde Coletiva, Rio de Janeiro, v. 5, n. 1, p. 163-177, 2000. Disponível em: http://goo.gl/SNlFWP. Acesso em: 10 jun. 2015.

BUSS, Paulo Marchiori; PELLEGRINI FILHO II, Alberto. A saúde e seus determinantes sociais. Physis: Revista de Saúde Coletiva, Rio de Janeiro, v. 17, n. 1, p. 77-93, 2007. Disponível em: http://goo. gl/sTWTcS. Acesso em: 10 jun. 2015.

CARRARA, Sergio; RUSSO, Jane; FARO, Livi. A política de atenção à saúde do homem no Brasil: os paradoxos da medicalização do corpo masculino. Physis: Revista de Saúde Coletiva, Rio de Janeiro, v. 19, n. 3, p. 659-678, 2009. Disponível em: http://goo.gl/etvQsx. Acesso em: 03 mar. 2016.

CARVALHO, Sergio Resende. Os múltiplos sentidos da categoria "empowerment" no projeto de promoção à saúde. Cadernos de Saúde Pública, Rio de Janeiro, v. 20, n. 4, p. 1088-1095, 2004. Disponível em: http://goo.gl/ljroAq. Acesso em: 17 maio 2016.

CONNELL, Robert. Políticas da masculinidade. Educação e Realidade, Florianópolis, v. 20, n. 2, p. 185-206, 1995. Disponível em: http://goo.gl/ j05lVz. Acesso em: 05 jun. 2015.

CONNELL, Robert; MESSERSCHMIDT, James. Masculinidade hegemônica: repensando o conceito. Estudos Feministas, Florianópolis, v. 21, n. 1, p. 241-282, 2013. Disponível em: https:// goo.gl/nAN7FO. Acesso em: 05 jun. 2015.

COSTA-ROSA, Abílio. Práticas de cura místicoreligiosas, psicoterapia e subjetividade contemporânea. Psicologia USP, São Paulo, v. 19, n. 4, p. 561-590, 2008. Disponível em: http://goo. gl/L4JHaL. Acesso em: 30 mar. 2016.

CZERESNIA, Dina. 0 conceito de saúde e a diferença entre prevenção e promoção. In: CZERESNIA, Dina; FREITAS, Carlos Machado de Freitas (org.). Promoção da saúde: conceitos, reflexões, tendências. Rio de Janeiro: Editora Fiocruz, 2003. p. 39-53.

CZERESNIA, Dina. Ações de promoção à saúde e prevenção de doenças: o papel da ANS. In: MONTONE, Januário; CASTRO, Antonio Joaquim Werneckde (org.). Regulação \& Saúde. Documentos técnicos de apoio ao Fórum de Saúde Suplementar de 2003. Vol. 3. Rio de Janeiro: Ministério da Saúde/ Agência Nacional de Saúde Suplementar, 2004. p. 211-240.

ESCOBAR, Maria del Pilar; PICO, Maria. Autocuidado de la salud em jóvenes universitarios. Revista Facultad Nacional de Salud Pública [enlinea], Bogotá, v. 31, n. 2, p. 178-186, 2013. Disponível em: 
http://goo.gl/DBn6Ns. Acesso em: 30 mar. 2016.

GAVIN, Rejane Ospedal Salomão; OLIVEIRA, Maria Helena Pessini de; SILVA, Gherardi-Dona da, Edilaine Cristina. Terapias alternativas complementares: uma visão do conhecimento dos acadêmicos de enfermagem. Ciência, Cuidado e Saúde, v. 9, n. 4, p. 760-765, 2010. Disponível em: http://goo.gl/ hwmM30. Acesso em: 18 ago. 2015.

GOMES, Romeu. A saúde do homem em foco. São Paulo: UNESP, 2010.

GOMES, Romeu. A saúde e a sexualidade do homem em revista. In: GOMES, Romeu. Sexualidade masculina, gênero e saúde. Rio de Janeiro: Editora FIOCRUZ, 2012. p. 99-113.

HEIDMANN, Ivonete Buss. Et al. Promoção à saúde: trajetória histórica de suas concepções. Texto Contexto Enfermagem, Florianópolis, v. 15, n. 2, p. 352-358, 2006. Disponível em: http://goo. gl/99vHC9. Acesso em: 18 ago. 2015.

HOFF, Tânia Maria Cezar. Publicidade: o corpo modificado. Comunicação, Mídia e Consumo, São Paulo, v. 1, n. 1, p. 52-62, 2004. Disponível em: http://goo.gl/X8tfRo. Acesso em: 05 jul. 2015.

KÜLKAMP, Irene. Et al. Aceitação de práticas nãoconvencionais em saúde por estudantes de medicina da Universidade do Sul de Santa Catarina. Revista Brasileira de Educação Médica, Rio de Janeiro, v. 31, n. 3, p. 229-235, 2007. Disponível em: http:// goo.gl/8vUtzK. Acesso em: 30 mar. 2016.

LAURENTI, Rui; JORGE, Maria Helena Prado de Melo; GOTLIEB, Sabina Lea Davidson. Perfil epidemiológico da morbi-mortalidade masculina. Ciência \& Saúde Coletiva, Rio de Janeiro, v. 10, n. 1, p. 35-46, 2005. Disponível em: http://goo.gl/Smdq9X. Acesso em: 04 mar. 2016.

MATOS, Ana PaulaSoaresde; SOUSA-ALBUQUERQUE, Carlos Manuel. Estilo de vida, percepção e estado de saúde em estudantes portugueses: influência da área de formação. International Journal of Clinical and Health Psychology, Espanha, v. 6, n. 3, p. 647-663, 2006. Disponível em: http://goo.gl/ Q9DvCu. Acesso em: 28 mar. 2016.

MINAYO, Maria Cecília de Souza. Saúde-doença: uma concepção popular da etiologia. Cadernos de Saúde Pública [online], Rio de Janeiro, v. 4, n. 4, p. 363-381, 1988. Disponível em: http://goo. gl/2SUQx1. Acesso em: 17 jun. 2016.

MINAYO, Maria Cecília de Souza. 0 desafio do conhecimento: pesquisa qualitativa em saúde. São
Paulo: Hucitec, 2010.

MORALES, Naiara Mendes; MIN, Li Shih; TEIXEIRA, João Eduardo Marten. Atitude de estudantes de medicina frente a terapias alternativas e complementares. Revista Brasileira de Educação Médica, Rio de Janeiro, v. 39, n. 2, p. 240-245, 2015. Disponível em: http://goo.gl/SqPUHL. Acesso em: 16 dez. 2015.

NAHAS, Markus Vinícius. 0 pentáculo do bem-estar. Boletim do Núcleo de Pesquisa em Atividade Física \& Saúde, Rio Grande do Sul, v. 2, n. 7, p. 0103, 1996. Disponível em: http://goo.gl/4dUHne. Acesso em: 10 mar. 2016.

NAHAS, Markus Vinícius; BARROS, Mauro de; FRANCALACCI, Vanessa. 0 pentáculo do bem-estar: base conceitual para avaliação do estilo de vida de indivíduos ou grupo. Revista Brasileira de Atividade Física \& Saúde, Rio Grande do Sul, v.5, n.2, p.48-59, 2000. Disponível em: https://goo.gl/ nM80rO. Acesso em: 10 mar. 2016.

PIETRUKOWICZ, Maria Cristina Leal Cypriano. Apoio social e religião: uma forma de enfrentamento dos problemas de saúde. 2001. 117f. Dissertação (Mestrado em Saúde Pública) Fundação Oswaldo Cruz (Fiocruz), Escola Nacional de Saúde Pública (ENSP), Rio de Janeiro, 2001.

PINHEIRO, Thiago Félix; COUTO, Maria Thereza. Homens, masculinidades e saúde: uma reflexão de gênero na perspectiva histórica. Caderno de História da Ciência, São Paulo, v. 4, n. 1, p. 5367, 2008. Disponível em: http://goo.gl/9pLvi0. Acesso em: 29 mar. 2016.

REIS, Jessyluce Cardoso. Religiosidade popular: o poder simbólico cultural e a interpretação do sagrado. Revista Mosaicum, n. 6, p. 67-76, 2007. Disponível em: https://revistamosaicum.org/ index.php/mosaicum/article/view/384/341. Acesso em: 29 mar. 2016.

ROCHA, Daniele Machado Pereira Rocha. Concepções, autopercepção e práticas ligadas à saúde e doença de estudantes universitários masculinos do bacharelado interdisciplinar em saúde da Universidade Federal da Bahia. 2017. 117f. Dissertação (Mestrado em Estudos Interdisciplinares sobre a Universidade) - Instituto de Humanidades, Artes e Ciências Professor Milton Santos, Universidade Federal da Bahia (UFBA), Salvador, 2017.

RODRIGUES, Janaina Furtado; RIBEIRO, Elaine Rossi. 0 homem e a mudança de pensamento em 
relação à sua saúde. Caderno Saúde e Desenvolvimento, Curitiba, v. 1, n. 1, p. 74-86, 2012. Disponível em: http://goo.gl/2GWMab. Acesso em: 03 mar. 2016.

SICOLI, Juliana Lordelo; NASCIMENTO, Paulo Roberto do. Promoção de saúde: concepções, princípios e operacionalização. Interface, Botucatu, SP, v. 7, n. 12, p. 91-112, 2003. Disponível em: http://goo.gl/IwgTLt. Acesso em: 18 ago. 2015.

SOUZA JUNIOR, Sergio Luís Peixoto; BIER, Anelise. A importância da atividade física na promoção de saúde da população infanto-juvenil. EFDeportes. com - Revista Digital, Buenos Aires, ano 13, n. 119, 2008. Disponível em: http://goo.gl/eJlVUH. Acesso em: 05 ago. 2015.

TROVÓ, Mônica Martins; SILVA, Maria Elúlia Paes da. Terapias alternativas/complementares - a visão do graduando de enfermagem. Revista Escola de Enfermagem, São Paulo, v. 36, n. 1, p. 80-87, 2002. Disponível em: http://goo.gl/gH4f6t. Acesso em: 17 dez. 2015.

VIDAL, Rogério Lima. Linguagem e saberes tradicionais africanos nas práticas de cura dos benzeiros na comunidade quilombola do mucambo. In: SEMINÁRIO INTERNACIONAL ACOLHENDO AS LÍNGUAS AFRICANAS (SIALA), 2012, Salvador. Anais... Salvador: SIALA, 2012.p. 1-15.

Recebido em: 18/03/2019

Revisado em: 20/07/2020

Aprovado em: 08/08/2020 